\title{
Editorial
}

\section{Un modelo de desarrollo científico en Costa Rica}

A lo largo de la historia, la medicina ha evolucionado constantemente. De la simple observación de una enfermedad se pasó a anotar los hallazgos clínicos en forma sistemática, con el fín de mejorar el tratamiento y la atención de los pacientes. Durante muchos años, resulto un orgullo para el médico hacer el diagnóstico de una dolencia utilizando solamente las manifestaciones clínicas de sus pacientes, y hasta implicaba un menosprecio a su categoría profesional ayudarse con el laboratorio o los rayos X. Sin, embargo ante los inminentes avances en técnicas diagnósticas y farmacológicas, tanto la clasificación de las enfermedades como los tratamientos concretos empezaron a hacerse de manera más científica.

Costa Rica no fue la excepción. Por mucho tiempo, y ante la imposibilidad de contar con aparatos sofisticados o técnicas modernas, el médico debió usar su aprendizaje para solucionar todas las situaciones a las que lo enfrentaba su trabajo diario. En muchas ocasiones, como me relataron algunos médicos ya fallecidos, su sentimiento permanente era de frustración, por no contar con medicamentos ni procedimientos adecuados que les permitieran clasificar adecuadamente las diferentes patologías infecciosas que por centurias atacaron al ser humano. Al prolongarse la vida del hombre comenzaron a aparecer patologías poco conocidas, o que tal vez no se mostraban anteriormente en la corta vida de los seres humanos. Todo esto hizo que las enfermedades se complicaran.

Aun así, como decía Teilhard de Chardin, en última instancia todo se une en un solo punto que él llama la ciencia de la naturaleza. O sea, que la clínica y las ayudas colaterales tienen un mismo objetivo: aliviar a los enfermos.

El desarrollo del análisis de las hemoglobinas anormales y de las enzimoeritropatías, es un estudio relativamente nuevo de nuestro país y merece una pequeña reflexión. Hace poco más de cincuenta años, en Costa Rica no había posibilidad de diagnosticar estas dolencias, menos decir si los pacientes eran homocigotos o heterocigotos para esos problemas. Sin embargo, el desarrollo sistemático de la ciencia, y sobre todo la calidad de los médicos e investigadores, hizo que ese tipo de pacientes se estudiaran con gran responsabilidad académica.

De ahí que la comunidad científica, dirigida por el Dr. German Sáenz Renauld, creara en 1980 el Centro de Investigación en Hemoglobinas Anormales y Trastornos Afines (CIHATA) de la Facultad de Microbiología de la Universidad de Costa Rica, al cual le cabe la potestad de haber descubierto varias hemoglobinas autóctonas y también diversas mutantes de las enzimas eritrocitarias. Este Centro cambió el concepto tradicional, que siempre consideró que las hemoglobinopatías presentaban anemia, y nunca policitemia, como fue encontrado en algunas variantes con gran afinidad por el oxígeno.

Gracias a esos trabajos y a la labor desplegada por la Asociación Costarricense de Hematología, los médicos de adultos y pediatras, acompañados siempre por microbiólogos especialistas, realizaron visitas a todos los centros hospitalarios del país para mejorar la atención y el diagnóstico de las hemoglobinopatías en Costa Rica.

En la actualidad, el CIHATA realiza técnicas de biología molecular, dando una nueva dimensión al estudio de las hemoglobinas anormales y también a las variantes enzimáticas eritrocitarias. De esta manera, es posible rastrear en la actualidad la procedencia de las mutaciones de la hemoglobina o de las enzimas, lo cual conlleva a una nueva perspectiva histórica y antropológica.

El ejemplo del desarrollo histórico del problema en cuestión, es digno de mencionarse, y debe llenarnos de orgullo que en nuestro país existan científicos de la talla del Dr. Sáenz, que no sólo crearon un Centro de Investigación, sino que formaron discípulos para continuar esa labor. En la actualidad, el Programa Nacional de Tamizaje Neonatal, uno de los mejores de América Latina, estudia la posibilidad de incluir dentro de sus estudios, el análisis de las hemoglobinas anormales, lo cual, dado la amplia cobertura de ese programa, diagnosticaría este problema desde el nacimiento, con claras ventajas para los pacientes y sus familiares.

ISSN 0001-6002/2005/47/4/172 Acta Médica Costarricense, (C2005 Colegio de Médicos y Cirujanos
Dr. Rafael Jiménez Bonilla

Jefe de la Unidad de Bioética e Investigación

Hospital Nacional de Niños "Dr. Carlos Sáenz Herrera". 